\title{
25
}

\section{Schedule Planning by Using Genetic Algorithms for Developing Educational Materials and Curricula}

\author{
Tsurayuki Kado and Seiichi Komiya ${ }^{\mathrm{b}}$ \\ a Computer Group, Hitachi Ltd., 27-18, Minami Oi 6-chome, Shinagawa-ku, Tokyo, 140, Japan \\ b Information-technology Promotion Agency, 3-1-38 Shibakoen, Minato-ku, Tokyo, 105, Japan
}

\begin{abstract}
The development and use of software products depends entirely on people. When many people make intellectual contributions to a group activity, individual ability and circumstances affect the overall quality, quantity, and schedule. It is not wise to plan time schedules manually, because the increasing number of participants will introduce many constraints.

The authors consider schedule planning for the cooperative development of educational materials and curricula an optimum combination problem and introduce solutions through the use of genetic algorithms. More specifically, this paper describes a method of schedule planning using genetic algorithms, and discusses the effectiveness of this approach.
\end{abstract}

Keyword Codes: I. 2. 8; K. 3. 1; K. 6.1

Keywords: Genetic Algorithms, Optimum Combination Problem, Generate and Test Method; Software Process Management, Schedule Planning

\section{INTRODUCTION}

Creating schedules for the development of educational materials and curricula is an old and new problem for educational managers. The problems are not as difficult when there are relatively few objective curricula and people involved. The complexity increases significantly with an increase in curricula and participants.

One of the most important constraints in the development of educational materials and curricula is whether or not the developers of the curriculum have the necessary skills and qualifications to create the required educational materials and curricula. For that reason, there are relatively few developers of educational materials in comparison with the number of courses that need to be developed.

Since people with specific skills or qualifications are in great demand, schedules for developing educational materials and curricula are typically planned to fit their schedules. These capable people must often be engaged in many different activities at the same time. In such situations, it is important to develop plans that limit the burden on specialized people and optimize use of available manpower. For this purpose it is important to consider the development of an advanced planning tool. 


\section{OUTLINE OF A MODEL WITH GENETIC ALGORITHMS}

There are five instructors who teach seven courses related to UNIX. Figure 1 shows an example of a curriculum. Figure 2 shows the skill level for each participant. Course $D$ is new, so the teachers must develop new educational materials for $\mathrm{D}$. Figure 2 also shows the procedure for developing the materials, lectures and exercises for class $\mathrm{D}$ and the possibility of each participant. The process is serial macroscopically, but it may be parallel microscopically. The managers leave the planning of lectures and exercises in the instructor's hands. In this model, there is no strict discipline on the order as in the Water Fall Model. Only the termination time of each process is given as a condition. If the beginning time of each course and its instructor's schedule are determined as in Figure 3, which development plan for the new class $\mathrm{D}$ is optimum under these constraints?

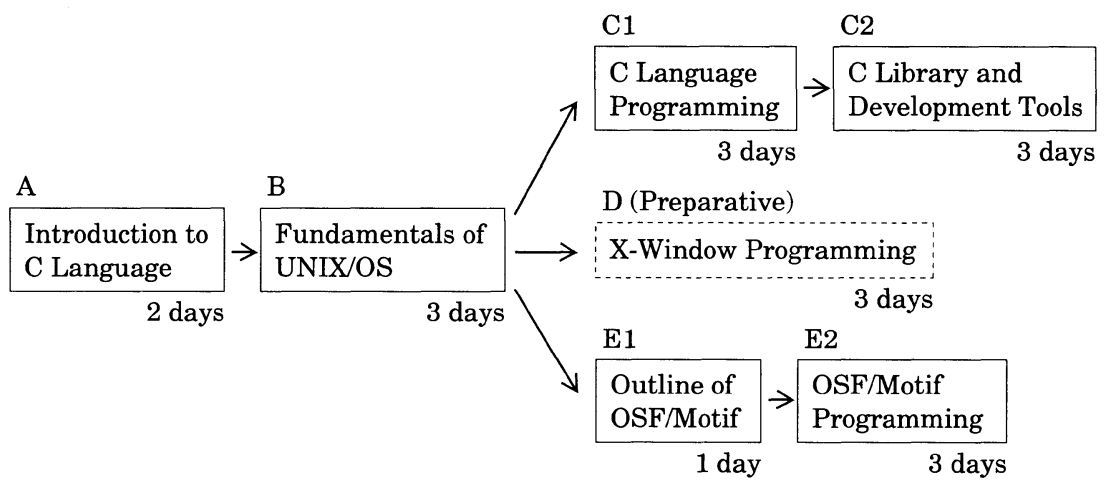

Figure $1:$ Example of a Curriculum

\begin{tabular}{|c|c|c|c|c|c|c|c|c|c|c|c|c|}
\hline \multirow{2}{*}{$\begin{array}{l}\text { Course } \\
\text { Instructor } \\
\text { (skill level) }\end{array}$} & \multirow{2}{*}{$\mathrm{A}$} & \multirow{2}{*}{$\mathrm{B}$} & \multirow{2}{*}{$\mathrm{C}$} & \multicolumn{8}{|c|}{$\mathrm{D}$} & \multirow{2}{*}{$\mathrm{E}$} \\
\hline & & & & RA & $\mathrm{CD}$ & $\mathrm{AU}$ & DD & $\begin{array}{l}\mathrm{DR} \\
\end{array}$ & LE & EX & EV & \\
\hline a (middle level) & 0 & & 0 & 0 & 0 & 0 & & & & 0 & 0 & \\
\hline b (middle level) & & 0 & & 0 & 0 & 0 & O & 0 & O & 0 & 0 & 0 \\
\hline c (high level) & 0 & & 0 & O & $\bigcirc$ & 0 & & 0 & 0 & 0 & 0 & \\
\hline d (high level) & & 0 & & 0 & 0 & 0 & O & O & 0 & 0 & 0 & 0 \\
\hline e (Group Leader) & 0 & 0 & 0 & 0 & 0 & 0 & O & 0 & 0 & 0 & 0 & O \\
\hline f (Manager) & & & & & & 0 & & & & & 0 & \\
\hline
\end{tabular}

RA : Requirement Analysis CD : Concept Design AU : Review \& Authorization $\mathrm{DR}:$ Design Review LE : Lecture EX : Exercises

DD : Detailed Design EV : Evaluation

Figure 2 : Skill Level of Participants 


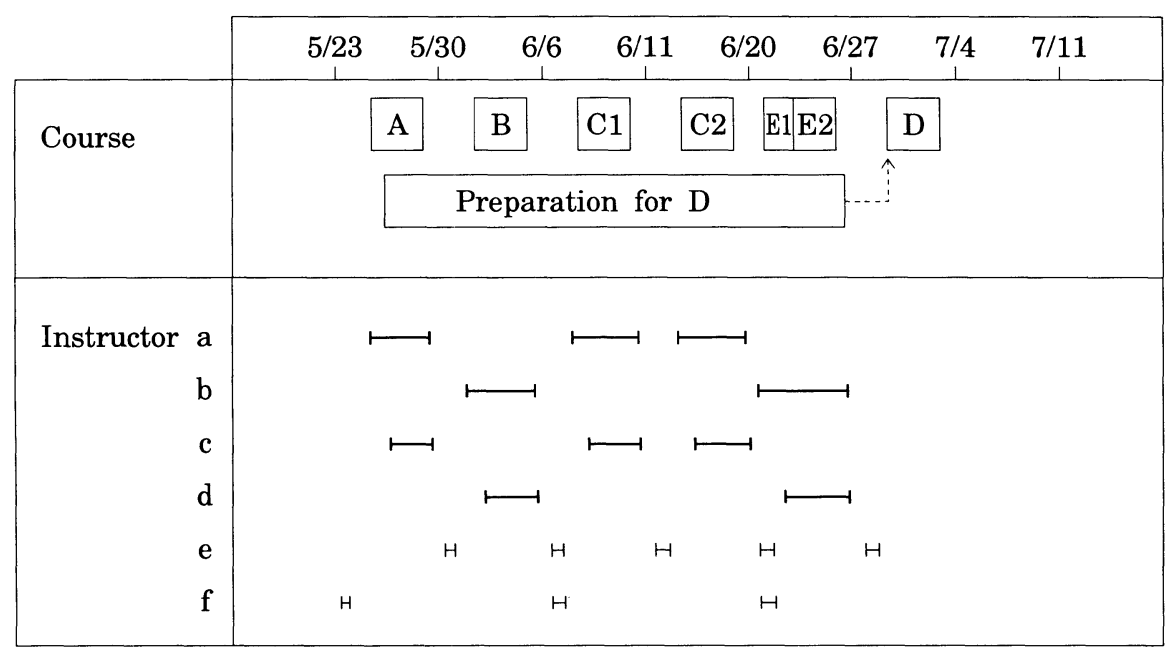

Figure 3 : Constraints on Schedule

The best method for solving this problem is to approve adaptation to constraints given after successively generating the conceivable combinations. We adapted Genetic Algorithms (GA method) as a kind of Generate and Test Method. We use the GA method which arose from the Genetic Operators Theory by J. H. Holland in 1975 for an Optimum Search Method.

In the GA method, the combinations of the working process and participants are indicated as a character set called a 'chromosome'. Each character corresponds to a 'gene'. At first, we give a random character set as the initial value.

After the model increases the chromosomes as 0th generation, it repeats the process of natural selection. At last, we can get the near optimum solutions at the $n$th generation. By natural selection, the model generates the dominant genetic operation by cross-over between the two chromosomes, and by mutation just as with animals and plants. So we can quickly get balanced effective solutions.

The first character set contains the work descriptions (RA, CD, AU, DD, DR, LE, EX, EV) and is named chromosome $\mathrm{X}$. This $\mathrm{X}$ becomes the basic axis of natural selection in the model. We call it the Basic Layer. The second character set (a, c, f, a, e, c, a, e) is named chromosome M. Each gene, a, c, ..., e, and gene RA, CD, .., EV are in an one-to-one correspondence. The characters $\mathrm{a}, \mathrm{b}, \mathrm{c}, \mathrm{d}, \mathrm{e}, \mathrm{f}$ indicate instructors. The chromosome $\mathrm{M}$ contains the human resources. We call it the Middle Layer. The structure of this Middle Layer (length of character set) is the same as that of the Basic Layer (eight digit in this model). We can select any character (gene) in the Middle Layer at will. Three instructors can take charge of the whole eight processes. There is no limit on the number of participants . All instructors in charge (namely all qualified people) must be put on record beforehand as in Figure 2. This is one of the constraints.

Similarly, the character set (wp, wp, wp, ws, wp, ws, ws, wp) indicates the chromosome L. wp and ws stand for the word processor and work station used for the work.

The chromosome L contains non-human resources. We called it the Lower Layer. 
Figure 4 indicates that instructor ' $a$ ' takes charge of the detailed design (DD), and that instructor ' $a$ ' uses the work station for this work. When three instructors take charge of the same process, the character 'a' changes to 'b', 'd' and 'e', and the variety of chromosome increases. Our model is built on three layers.

The three layers seem to be limited because of complexity, however there is no restriction on the number of layers.

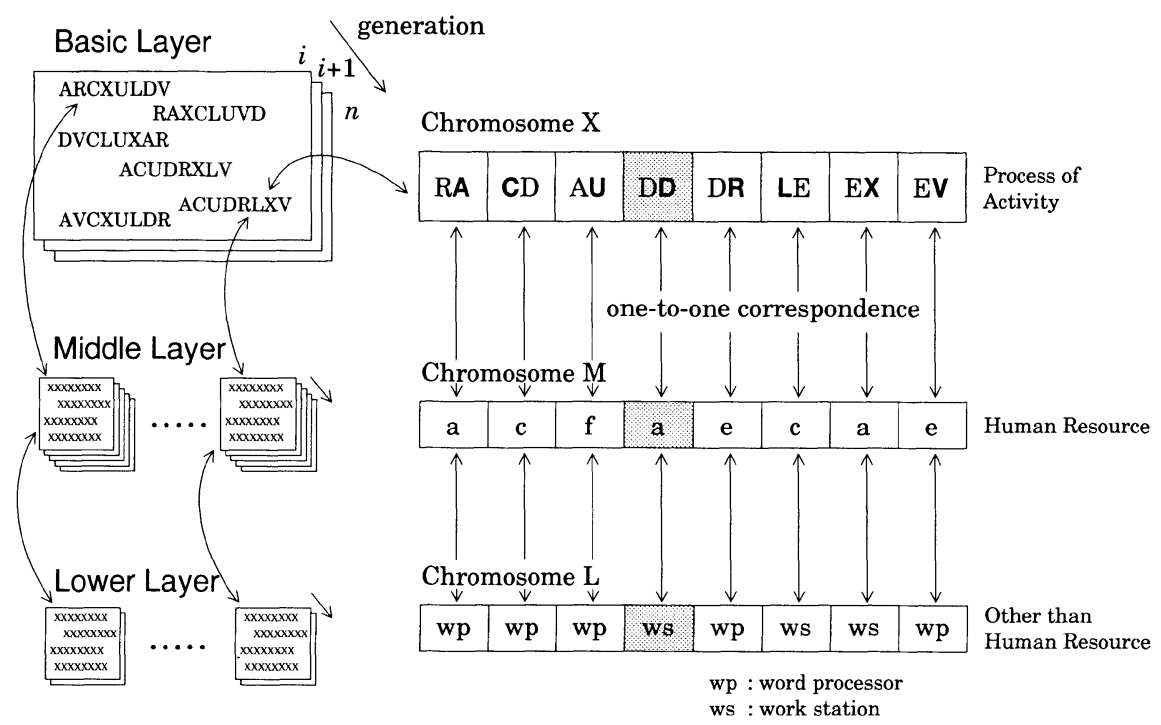

Figure $4:$ Concept of GA Model

The software production model has few significant character sets of the Basic Layer. In our model, the production process in Figure 2 is basic, to exchange mutually from LE into EX is only the significant process. However we allow variation as parallel processing between CD and DD, and between LE and EX. On the contrary, there are many chromosomes in the Middle Layer and the Lower Layer. If five instructors ' $a$ ', 'b', 'c', 'd' and 'e' have the same degree of ability, they can take charge of the whole process. Even if we provide the constraints as in Figure 2 in our experiment, we are worried about enormous combinations. The solutions which survive the constraints become candidates for the optimum solutions.

When a combination (chromosome $\mathrm{X}_{i}$ ) of instructors in charge is decided, the total necessary days $\left(\mathrm{Y}_{i}\right)$ for the job depend on each instructor's schedule. If our object is to shorten the days, we can replace this problem with the minimum solution problem.

At first, when we set a combination $\mathrm{X}_{0}$ as the initial value, we can get $\mathrm{Y}_{0}$. The model permits the initial values. Then the model generates $X_{1}$ by changing the genes in $X_{0}$, the model gets $Y_{1}$. At last, the model selects the near minimum solutions by repeating this operation. We introduced the concept of natural selection as cross-over and mutation instead of the random number generation method when the model generates the combina- 
Present Generation
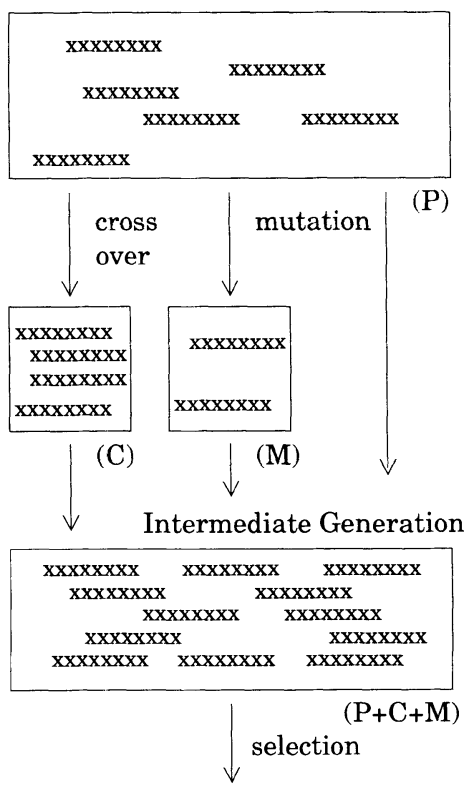

\begin{tabular}{|c|c|}
\hline \multicolumn{2}{|c|}{ Next Generation } \\
\hline & $\operatorname{xxxxxxxx}$ \\
\hline \multicolumn{2}{|c|}{$\operatorname{xxxxxxxx}$} \\
\hline $\mathrm{xxxxxxxx}$ & $\operatorname{xxxxxxxx}$ \\
\hline
\end{tabular}

(P)

Figure 5 : Process to Next Generation

tions. So this approach is called the GA method. Our model is named 'DROSOPHILA' because of the eight genes. DROSOPHILA is a genus of small flies used in laboratory experiments in heredity.

Figure 5 shows the mechanism of evolution of generation. There are $\mathrm{P}$ pieces of parent chromosome. DROSOPHILA generates $\mathrm{C}$ pieces of child by cross-over, and $\mathrm{M}$ pieces of child by mutation respectively. Then DROSOPHILA generates $(\mathrm{P}+\mathrm{C}+\mathrm{M})$ pieces of chromosome as the intermediate generation. Afterwards, DROSOPHILA selects the new $\mathrm{P}$ pieces of a dominant chromosome as the next generation by the select operation. A dominant chromosome means a chromosome with a high survival rate.

In the case of the minimum solution of $\mathrm{Y}=\mathrm{f}(\mathrm{X})$, a survival rate of the chromosomes which remain at the next generation is represented by the expression $\left(1-\mathrm{f}_{i} / \Sigma \mathrm{f}_{i}\right) /(\mathrm{n}-1)$

Figure 6 shows the search mechanism of GA. We can recognize that the candidates of the optimum solution boil down with the progress of generation. 


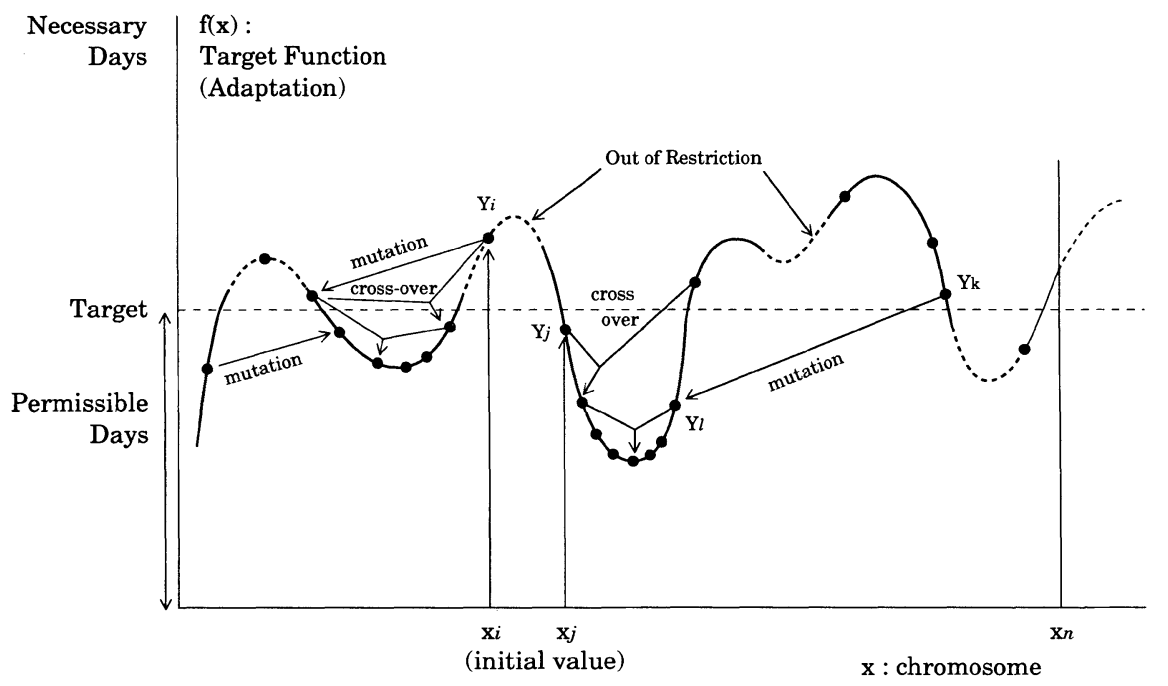

Figure 6 : Search Mechanism by GA

\section{OUTPUT, EVALUATION AND CONCLUSION}

Figure 7 and Figure 8 show two samples of desirable schedules for development of the new course $\mathrm{D}$.

The release from the complicated desk work by using the planning tool DROSOPHILA has great significance. The results went beyond our expectations, and are listed below :
(1) short time selection of the desirable solutions
(2) dissolution of the local solutions by preconception
(3) little influence created by fluctuating input data
(4) easy scrap and build

The GA method is certainly 'Robustness' as Holland has suggested. 


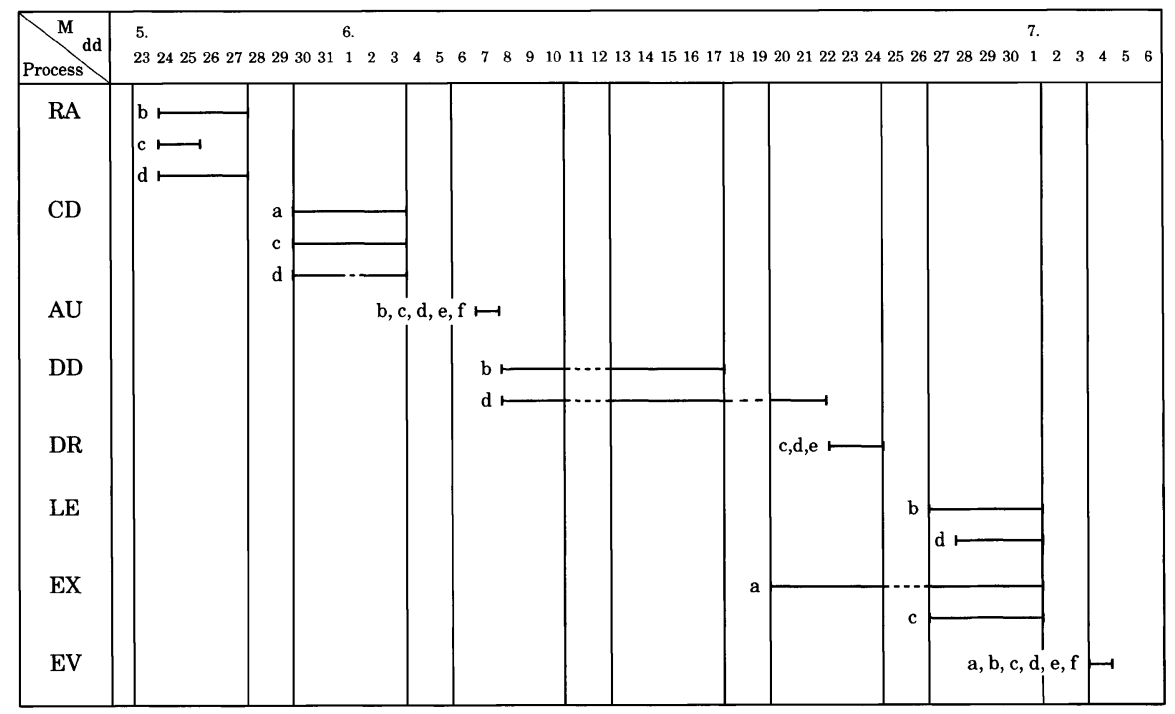

Figure 7 : Output Image (One Solution)

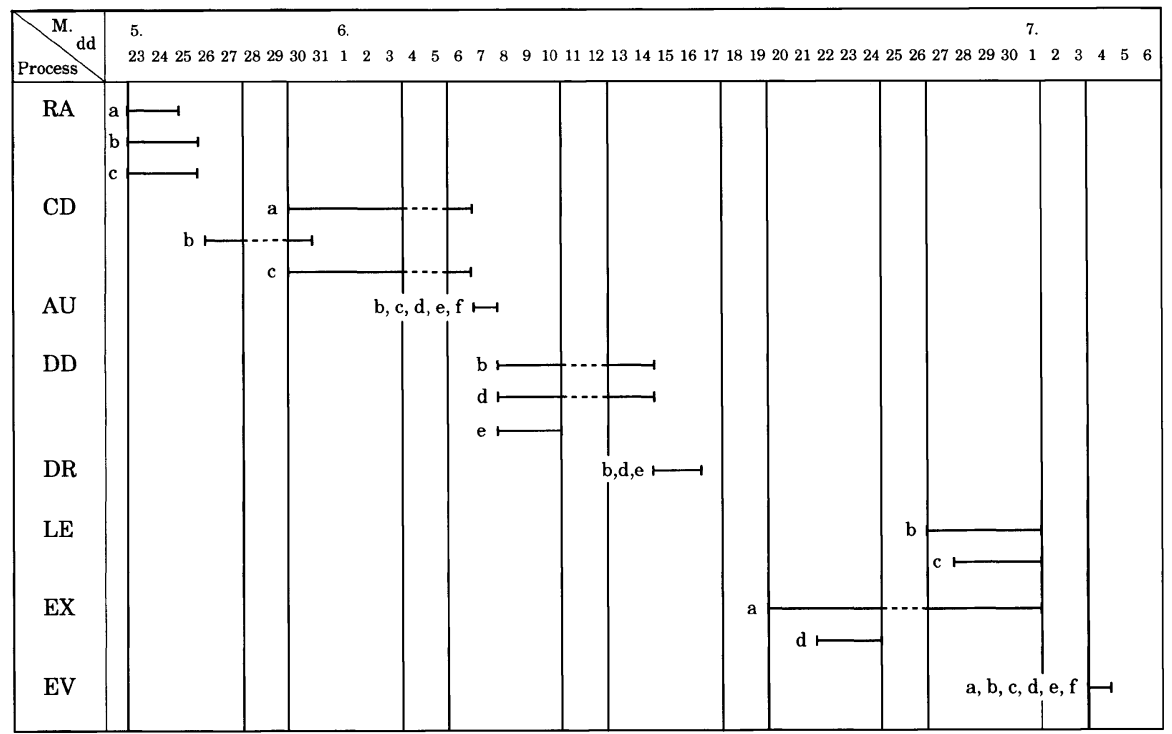

Figure 8 : Output Image (Another Solution) 


\section{ACKNOWLEDGMENTS}

The authors would like to thank Prof. Sam Stern (Oregon State Univ.), Miss Chieko Hashimoto and Mr.Scott K. Bachmann (Hitachi Ltd.) for their useful comments and their help in the editing of this paper.

\section{REFERENCES}

1. J. H. Holland, Adaptation in Natural and Artificial Systems, Univ. of Michigan Press, 1975, MIT Press, 1992.

2. D.E. Goldberg, Genetic Algorithms in Search, Optimization, and Machine Leaning, Addison Wesley Publishing Co. Inc., 1989

3. N.Sawabe and S. Komiya et al., Schedule Planning for Software Development with Genetic Algorithms, 1993 